\title{
FRACTIONAL INTEGRATION IN ORLICZ SPACES
}

\author{
ROBERT SHARPLEY
}

\begin{abstract}
Fractional integration and convolution results are given for Orlicz spaces using an inequality earlier developed for $\Lambda_{\alpha}(X)$ spaces which generalize Lorentz $L(p, q)$ spaces. The extension problem for convolution operators encountered previously by other authors is almost entirely avoided.
\end{abstract}

1. Introduction. O'Neil has shown that fractional integration and hence convolution theorems hold both for Lorentz $L(p, q)$ spaces [8] and by different methods for Orlicz spaces [9]. An inequality utilizing the weak interpolation for operators was given in [7] from which fractional integration theorems followed for the spaces $\Lambda_{\alpha}(X)$ which generalize the $L(p, q)$ spaces. This same inequality is used here to prove the corresponding theorem for Orlicz spaces. The main result of this paper avoids "endpoint estimates", but we hope to return to this point at a later time.

A function $A$ on $[0, \infty)$ is called a Young's function [9] if it is nondecreasing, convex, left continuous, satisfies $A(0)=0$, but is not identically zero. The Orlicz space $L_{A}$ is the Banach space of all locally integrable functions for which the Luxemburg norm

$$
\|f\|_{A}=\inf \left\{k \mid \int A(|f(x)| / k) d \mu(x) \leq 1\right\}
$$

is finite [3]. For simplicity, we shall restrict ourselves to the line $R$ with Lebesgue measure. If we set $A(x)=x^{p} / p$, then we obtain the $L^{p}$ spaces.

If $A$ is a Young's function, then we define its inverse by $A^{-1}(y)$ $=\inf \{x \mid A(x)>y\}$, where inf $\phi=\infty \cdot A^{-1}$ is a nondecreasing, right continuous function satisfying the conditions that $A^{-1}(x) / x$ is nondecreasing and

$$
A\left(A^{-1}(x)\right) \leq x \leq A^{-1}(A(x)) .
$$

If for every $\delta>1$ there is a constant $c$ so that $A(\delta x) \leq c A(x)$, then $A$ is said to satisfy the $\left(\delta_{2}, \Delta_{2}\right)$ condition.

If $f$ is a locally integrable function on $R$, then the distribution function for $|f|$ is defined by $\mu_{|f|}(t)=m(s|| f(s) \mid>t)$, where $m$ is Lebesgue measure. Two functions $f$ and $g$ are called equimeasurable if $\mu_{|f|}=\mu_{|g|}$. It is not hard to see then that $L_{A}$ is rearrangement invariant, i.e. equimeasurable functions $f$ and $g$ have the same norm in $L_{A}$. The decreasing rearrangement of a locally integrable function $f$ is the right continuous inverse of $\mu_{|f|}$ and is denoted by

Received by the editors April 14, 1975.

AMS (MOS) subject classifications (1970). Primary 26A33, 42A96; Secondary 46E30, 46E35.

Key words and phrases. Fractional integration, convolution, Orlicz spaces, decreasing rearrangement.

(c) American Mathematical Society 1976 
$f^{* *}$. The averaged rearrangement of $f$ is defined by $f^{* *}(t)=\int_{0}^{t} f^{*}(s) d s / t$, and it follows that $f^{*}(t) \leq f^{* *}(t)$.

The fundamental function $\varphi_{A}(t)$ of the space $L_{A}$ is the norm of the characteristic function of the interval $(0, t)$. A straightforward computation shows that $\varphi_{A}(t)=1 / A^{-1}(1 / t)$ and so $\varphi_{A}$ is a concave nondecreasing function on $(0, \infty)$ satisfying $\varphi_{A}(0)=0$. We define $\varphi_{A^{\prime}}(t)=t / \varphi_{A}(t)$. The indices [2], [5] of an Orlicz space are defined by

$$
\underline{\gamma}_{A}=\lim _{t \rightarrow 0} \theta(t), \quad \bar{\gamma}_{A}=\lim _{t \rightarrow \infty} \theta(t)
$$

where

$$
\theta(t)=\log \left(\sup _{s>0} \frac{\varphi_{A}(s t)}{\varphi_{A}(s)}\right) / \log t .
$$

It can be shown that $0 \leq \underline{\gamma}_{A} \leq \bar{\gamma}_{A} \leq 1$. When $A(x)=x^{p} / p$, then $\underline{\gamma}_{A}=\bar{\gamma}_{A}$ $=1 / p$. The reciprocals of these are the familiar exponents developed in [6]. By Lemma (5.9) of [2], $\underline{\gamma}_{A}>0$ is an equivalent condition on $A$ to the $\left(\delta_{2}, \Delta_{2}\right)$ condition. It is not difficult to see that $\bar{\gamma}_{A}<1$ is equivalent to $A^{\prime}$, the complementary Young's function determined by the fundamental function $\varphi_{A^{\prime}}(t)$, satisfying the $\left(\delta_{2}, \Delta_{2}\right)$ condition.

2. Convolution and special operators. A bilinear operator $T$ is called a convolution operator [9] if the following three conditions hold: (i) $\|T(f, g)\|_{1}$ $\leq\|f\|_{1}\|g\|_{1}$, (ii) $\|T(f, g)\|_{\infty} \leq\|f\|_{1}\|g\|_{\infty}$, (iii) $\|T(f, g)\|_{\infty} \leq\|f\|_{\infty}\|g\|_{1}$ where \|\|$_{1}$ and \|\|$_{\infty}$ denote the norms in $L^{1}$ and $L^{\infty}$, respectively. It is understood that $T$ is defined for a pair $(f, g)$ only when the existence is forced by relations (i) through (iii).

Special operators were defined in [2] in order to study the Hilbert transform. We shall need several of the properties of these operators for what follows. Define $P$ and $P^{\prime}$ by

$$
P(g)(t)=\int_{0}^{t} g(s) d s / t, \quad P^{\prime}(g)(t)=\int_{t}^{\infty} g(s) d s / s .
$$

Likewise, define the operator $S$ by

$$
S(g)=\varphi_{A^{\prime}} P(g)+P^{\prime}\left(g \varphi_{A^{\prime}}\right) .
$$

Proposition (2.1) (SEe [2]). Suppose that B and $C$ are Young's functions. $A$ necessary and sufficient condition that $P$ is a bounded operator on $L_{B}$ is that $\bar{\gamma}_{B}<1$. Dually, $\underline{\gamma}_{C}>0$ is necessary and sufficient for $P^{\prime}$ to be bounded on $L_{C}$.

We shall only use the sufficiency of this result which is not too hard to see. For example, in order to show $P$ is bounded on $L_{B}$, we represent $P(g)$ by $\int_{0}^{1} E_{t}(g)(s) d s$ where $E_{t} g(s)=g(s t)$ and then we notice that $\|P\|_{B}$ $\leq \int_{0}^{1}\left\|E_{1 / s}\right\|_{B} d s$. But

$$
\left\|E_{1 / s}\right\|_{B}=\sup _{r>0}\left\{\varphi_{B}(r s) / \varphi_{B}(r)\right\} \leq \text { const } s^{-\beta}, \quad \text { all } 0 \leq s \leq 1,
$$

for some $\beta<1$, since $\bar{\gamma}_{B}<1$. Hence $\|P\|_{B}<\infty$. 
The Lorentz space $M(A)$ [4] corresponding to $L_{A}$ is the Banach space of locally integrable functions $f$ such that

$$
\|f\|_{M(A)}=\sup _{t}\left\{f^{* *}(t) \varphi_{A}(t)\right\}
$$

is finite. In [10] this space was denoted $M\left(L_{A}\right)$. If $A(x)=x^{p} / p$, then $M(A)$ is just "weak $L^{p}$ ". We only require the following property of this space, namely

$$
\|f\|_{M(A)} \leq\|f\|_{A} .
$$

We now state the main inequality for convolution operators mentioned in the introduction.

Proposition (2.3) (SEE [7]). If $f \in M(A)$ and $g$ satisfies the condition that $S\left(g^{*}\right)\left(t_{0}\right)$ is finite for some $t_{0}$, then $T$ can be uniquely extended to be defined for the pair $(f, g)$ and

$$
T(f, g)^{*} \leq 2\|f\|_{M(A)} S\left(g^{*}\right) .
$$

Remark (2.5). The reader should notice that the quantity $\|f\|_{M(A)} S\left(g^{*}\right)$ is very similiar to the expression

$$
t f^{* *}(t) g^{* *}(t)+\int_{t}^{\infty} f^{*}(s) g^{*}(s) d s
$$

used in [8], [1], [2]. In fact, if $f^{* *}(t)$ and $f^{*}(t)$ are replaced in the second expression by $\|f\|_{M(A)} / \varphi_{A}(t)$, then we obtain the first expression. In a sense, $1 / \varphi_{A}(t)$ can be thought of as the largest function of norm one in $M(A)$, so lon: as $\bar{\gamma}_{A}<1$. It should also be noted here that Proposition (2.3) seems to avoid the extension problem that has plagued writers in the past [1], [12].

3. Main result. In order to arrive at the correct estimates involving Young's functions, we need the following lemma which is a modification of the "Generalized Young's Inequality" (Lemma (2.4) of [9]). Below we use $c$ to denote an absolute constant, not necessarily the same in all the formulas in which it occurs.

Lemma (3.1). Suppose $A, B$, and $C$ are Young's functions such that $C$ satisfies the $\left(\delta_{2}, \Delta_{2}\right)$ condition, then the following are equivalent

$$
\begin{aligned}
\varphi_{C}(s) s & \leq c \varphi_{A}(s) \varphi_{B}(s), \\
A^{-1}(t) B^{-1}(t) & \leq c t C^{-1}(t), \\
C\left(B^{-1}(B(y)) t A^{-1}(1 / t)\right) & \leq c B(y) \quad \text { when } B(y) \leq 1 / t .
\end{aligned}
$$

Proof. By the definitions it is obvious that (3.2) and (3.3) are equivalent. We show that condition (3.3) implies (3.4). Since $B(y) \leq 1 / t$ and $A^{-1}(x) / x$ is nondecreasing, we have

$$
\begin{aligned}
C\left(B^{-1}(B(y)) t A^{-1}(1 / t)\right) & \leq C\left(B^{-1}(B(y)) A^{-1}(B(y)) / B(y)\right) \\
& \leq C\left(c C^{-1}(B(y))\right) \leq \text { const } B(y) .
\end{aligned}
$$


The last two inequalities are valid since (3.3) holds and $C$ satisfies the $\left(\delta_{2}, \Delta_{2}\right)$ condition. That (3.4) implies (3.3) is seen by setting $t=1 / B(y)$.

By the definition of $\varphi_{A^{\prime}}(t)$ and relation (1.2) we have

COROLLARY (3.5). If the assumptions of Lemma (3.1) hold along with relation (3.2), then we have

$$
C\left(y \varphi_{A^{\prime}}(t)\right) \leq c B(y) \text { when } B(y) \leq 1 / t .
$$

Lemma (3.7). If $\|g\|_{B} \leq c$, then $B\left(g^{*}(t) / c\right) \leq 1 / t$.

Proof. By property (2.2) and the fact $\|g\|_{B} \leq c$, we get

$$
g^{*}(t) / c \leq g^{* *}(t) / c \leq 1 / \varphi_{B}(t)=B^{-1}(1 / t) .
$$

Applying $B$ to both sides of this inequality, we obtain

$$
B\left(g^{*}(t) / c\right) \leq B\left(B^{-1}(1 / t)\right) \leq 1 / t
$$

Theorem (3.8). Suppose $A, B$, and $C$ are Young's functions for which $\bar{\gamma}_{B}<1$ and $\underline{\gamma}_{C}>0$ and

$$
s \varphi_{C}(s) \leq c \varphi_{A}(s) \varphi_{B}(s),
$$

then each convolution operator has a unique extension to $M(A) \times L_{B}$ such that $T(f, g)$ belongs to $L_{C}$ whenever $f \in M(A)$ and $g \in L_{B}$; moreover,

$$
\|T(f, g)\|_{C} \leq c\|f\|_{M(A)}\|g\|_{B} .
$$

Proof. We assume without loss of generality that $\|g\|_{B}=1$. By Proposition (2.1)

$$
\left\|P\left(g^{*}\right)\right\|_{B} \leq c_{1}\left\|g^{*}\right\|_{B}=c_{1}\|g\|_{B}=c_{1}
$$

and

$$
\left\|P^{\prime}\left(g^{*} \varphi_{A^{\prime}}\right)\right\|_{C} \leq c_{2}\left\|g^{*} \varphi_{A^{\prime}}\right\|_{C} .
$$

Applying Lemma (3.7) to $P\left(g^{*}\right)$ and (3.10), we get that $B\left(P\left(g^{*}\right)(t) / c_{3}\right) \leq 1 / t$ where $c_{3}=c_{1}+\varepsilon$. Using Corollary (3.5) with $y=P\left(g^{*}\right)(t) / c_{3}$ and integrating we get

$$
\int C\left(P\left(g^{*}\right)(t) \varphi_{A^{\prime}}(t) / c_{3}\right) d t \leq \int B\left(P\left(g^{*}\right)(t) / c_{3}\right) d t \leq 1
$$

by the definition of the norm (1.1). Hence we have

$$
\left\|P\left(g^{*}\right) \varphi_{A^{\prime}}\right\|_{C} \leq c_{1}\|g\|_{B} .
$$

Similarly, by Lemma (3.7) we have that $B\left(g^{*}(t) /(1+\varepsilon)\right) \leq 1 / t$ so Corollary (3.5) with $y=g^{*}(t) /(1+\varepsilon)$ after integration yields

$$
\int C\left(g^{*}(t) \varphi_{A^{\prime}}(t) /(1+\varepsilon)\right) d t \leq \int B\left(g^{*}(t) /(1+\varepsilon)\right) d t \leq 1
$$


and so there holds

$$
\left\|g^{*} \varphi_{A^{\prime}}\right\|_{C} \leq\|g\|_{B}
$$

Combining inequalities (3.11) and (3.13), we obtain

$$
\left\|P^{\prime}\left(g^{*} \dot{\varphi}_{A^{\prime}}\right)\right\|_{C} \leq c_{2}\|g\|_{B} .
$$

But this together with (3.12) gives

$$
\left\|S\left(g^{*}\right)\right\|_{C} \leq c\|g\|_{B} .
$$

Hence $S\left(g^{*}\right)$ is finite almost everywhere and we can use Proposition (2.3) to get that $T(f, g)$ is defined for each $f \in M(A)$. By (2.4) we obtain

$$
\|T(f, g)\|_{C} \leq 2\|f\|_{M(A)}\left\|S\left(g^{*}\right)\right\|_{C} \leq c\|f\|_{M(A)}\|g\|_{B} .
$$

TheOREM (3.14). Suppose $A, B$, and $C$ are Young's functions but condition (3.9) does not hold, then there exist functions $f \in M(A)$ and $g \in L_{B}$ such that $f * g \notin M(C)$.

Proof. We denote by $\Lambda(A)$ the Banach space of all locally integrable functions such that

$$
\|f\|_{\Lambda(A)}=\int_{0}^{\infty} f^{*}(s) d \varphi_{A}(s)
$$

is finite. It is not hard to see [9], [10] that for each $f \in \Lambda(A)$

$$
\|f\|_{A} \leq\|f\|_{\Lambda(A)}
$$

so $\Lambda(A)$ is continuously embedded in $L_{A}$. We show in fact that

$$
\Lambda(A) * \Lambda(B) \nsubseteq M(C) .
$$

Since relation (3.9) does not hold, there is a sequence $\left\{s_{n}\right\}$ such that

$$
s_{n} \varphi_{C}\left(s_{n}\right) \geq c_{n} \varphi_{A}\left(s_{n}\right) \varphi_{B}\left(s_{n}\right)
$$

where $c_{n}=2^{4 n}$. Define $f$ and $g$ by

$$
f(t)=\sum_{1}^{\infty} c_{n}^{-1 / 4} / \varphi_{A}\left(s_{n}\right) \chi_{\left[-s_{n} / 2, s_{n} / 2\right]}(t)
$$

and

$$
g(t)=\sum_{1}^{\infty} c_{n}^{-1 / 4} / \varphi_{B}\left(s_{n}\right) \chi_{\left[-s_{n} / 2, s_{n} / 2\right]}(t)
$$

In this case we have $\|f\|_{\Lambda(A)}=\|g\|_{\Lambda(B)}=1$, since $f$ and $g$ decrease symmetrically. But

$$
(f * g)(x) \geq \frac{1}{4} \sum_{1}^{\infty} c_{n}^{-1 / 2} \frac{s_{n}}{\varphi_{A}\left(s_{n}\right) \varphi_{B}\left(s_{n}\right)} \chi_{\left[-s_{n} / 2, s_{n} / 2\right]}(x)
$$


so

$$
\begin{aligned}
\|f * g\|_{M(C)} & \geq \sup _{m}\left(\frac{c_{m}^{-1 / 2} s_{m}}{4 \varphi_{A}\left(s_{m}\right) \varphi_{B}\left(s_{m}\right)} \varphi_{C}\left(s_{m}\right)\right) \\
& \geq \sup _{m} \frac{1}{4} c_{m}^{1 / 2}=\infty .
\end{aligned}
$$

ReMARK (3.15). By private correspondence M. Milman has sent the author a proof of Theorem (3.14) involving the Orlicz spaces themselves rather than the spaces $\Lambda$ and $M$. The proof follows closely O'Neil's result in this direction for product operators [9].

Combining Theorems (3.8) and (3.14), we obtain

Main Result. Suppose $A, B$, and $C$ are Young's functions such that $B^{\prime}$, the complementary Young's function for $B$, and $C$ satisfy the $\left(\delta_{2}, \Delta_{2}\right)$ condition. A necessary and sufficient condition that each convolution operator have a unique extension mapping $M(A) \times L_{B}$ into $L_{C}$ is that

$$
\varphi_{C}(s) s \leq c \varphi_{A}(s) \varphi_{B}(s)
$$

for some constant $c$.

4. Examples and further results. In this section we mention a related theorem and compare the results of this paper to those of O'Neil's. A discussion of the case $\underline{\gamma}_{C}=0$ is also included.

By the same methods employed in [9], duality and associate spaces, the following theorem is easily obtained.

THEOREM (4.1). If $A, B$, and $C$ are Young's functions such that $\bar{\gamma}_{A}<1$, $\bar{\gamma}_{B}<1$, and condition (3.9) holds, then $L_{A} * L_{B} \subseteq \Lambda(C)$. In fact, it is not hard to generalize this argument to rearrangement invariant Banach function spaces.

It is well known (p. 119 of [11]) that the conditions $\bar{\gamma}_{B}<1$ and $\underline{\gamma}_{C}>0$ in the Main Result are necessary if all the Young's functions are powers.

We can show that if $L_{C}=L^{\infty}$ and $B$ is any Young's function such that $\underline{\gamma}_{B}>0$, then selecting $A$ equal $B^{\prime}$, condition (3.9) holds, but $M(A) * L_{B}$ $\nsubseteq L_{C}$. However, the condition $\underline{\gamma}_{C}>0$ in Theorem (3.8) is not necessary in general. In [14] it is shown that fractional integration on $[0,2 \pi)$ holds for $A(x)=x^{p^{\prime}}, B(x)=x^{p}$, and $C(x)=e^{\lambda x^{p^{\prime}}} / \Lambda$ where $1 / p+1 / p^{\prime}=1$. The extrapolation argument given there holds for any convolution operator.

In [9, Theorem 4.7] it was shown that a fractional integration theorem holds for $\tilde{C}(x)$ defined by

$$
\tilde{C}^{-1}(x)=\int_{0}^{x} \frac{A^{-1}(t) B^{-1}(t)}{t^{2}} d t
$$

when $\tilde{C}$ exists and $B^{\prime}$ satisfies the $\left(\delta_{2}, \Delta_{2}\right)$ condition. By the definition one can show that if $\underline{\gamma}_{C}>0$ and $C$ satisfies (3.9), then $\tilde{C}$ exists and $\varphi_{C}(s) \leq c \varphi_{\tilde{C}}(s)$. This shows that Theorem (3.8) is weaker than O'Neil's.

A natural question which arises is: given $A$ and $B$, how can one construct a $C$ satisfying (3.9)? Define $C^{*}$ by the relation 


$$
\varphi_{C^{*}}(x)=\inf _{t \geq x} \frac{\varphi_{B}(t) \varphi_{A}(t)}{t} .
$$

If $\varphi_{C^{*}}$ is not identically zero on any interval, then $C^{*}$ exists and is equivalent (up to constants) to a Young's function. M. Milman has pointed out to the author that a necessary and sufficient condition that $C^{*}$ exist is that $A^{\prime}(x) \leqslant B(c x)$ for small values of $x$ (or symmetrically $B^{\prime} \leqslant A(c x)$ ). This is easily seen by taking inverses on both sides of the indicated inequality. If $C$ is any Young's function which satisfies (3.9), then

$$
\varphi_{C}(s) \leqslant c \varphi_{C^{*}}(s) .
$$

In particular, $\tilde{C}$ satisfies (3.9) so it must satisfy (4.2). Using the operators $P$ and $P^{\prime}$ one can show that if $\bar{\gamma}_{C^{*}}<1$, then $\underline{\gamma}_{C^{*}}>0$ is necessary and sufficient for $\tilde{C}$ and $C^{*}$ to be equivalent Young's functions. Zygmund's example shows that fractional integration theorems may hold even if $\tilde{C}$ does not exist. Professor O'Neil has pointed out to the author that Zygmund's example may be modified to give an example on the interval $[0,2 \pi)$ of a fractional integration theorem for $\tilde{C}$ but not for $C^{*}$. Let $\varphi_{A}(t)=\varphi_{B}(t)=\max \left(t, t^{\frac{1}{2}}\right)$, then $\varphi_{C^{*}}(t)$ $=1$ and $\varphi_{\tilde{C}}(t)=1 /(1+\ln (1 / t))$ for $0<t<1$. By the earlier arguments in this section we have $M(A) * L_{B} \nsubseteq L_{C^{*}}$ since $\underline{\gamma}_{B}=\frac{1}{2}>0$ and $L_{C^{*}}=L^{\infty}$, but by Zygmund's theorem with $p=2$ and $C(x)=e^{\lambda x^{2}} / \Lambda$ there must hold

$$
T: M(A) \times L_{B} \rightarrow L_{C} \subseteq L_{\tilde{C}}
$$

since $C$ dominates $\tilde{C}$ for large values. On the other hand, letting $\varphi_{A}(t)=t$ and $\varphi_{B}(t)=1$, it is easy to see that $\tilde{C}$ does not exist and $L_{C^{*}}=L^{\infty}$; but by the definition of convolution operator $T: L^{1} \times L^{\infty} \rightarrow L^{\infty}$. Since $M(A)=L_{A}$ in this case, we see that a theorem may hold for $C^{*}$ when $\tilde{C}$ does not exist.

\section{REFERENCES}

1. A. P. Blozinski, On a convolution theorem for $L(p, q)$ spaces, Trans. Amer. Math. Soc. 164 (1972), 255-265.

2. D. W. Boyd, The Hilbert transform on rearrangement-invariant spaces, Canad. J. Math. 19 (1967), 599-616. MR 35 \#3383.

3. M. A. Krasnosel'skiř and Ja. B. Rutickiĭ, Convex functions and Orlicz spaces, Fizmatgiz, Moscow, 1959; English transl., Noordhoff, Groningen, 1961. MR 21 \# 5144; 23 \# A4016.

4. G. G. Lorentz, Some new functional spaces, Ann. of Math. (2) 51 (1950), 37-55. MR 11, 442.

5. G. G. Lorentz and T. Shimogaki, Majorants for interpolation theorems, Publ. Ramanujan Inst. No. 1 (1968/69), 115-122. MR 43 \#954.

6. W. Matuszewska and W. Orlicz, On certain properties of $\varphi$-functions, Bull. Acad. Polon. Sci. Sér. Sci. Math. Astronom. Phys. 8 (1960), 439-443. MR 23 \# A3454. .

7 M. Milman and R. C. Sharpley, Convolution theorems for $\Lambda_{\alpha}(X)$ spaces (preprint).

8. R. O'Neil, Convolution operators and $L(p, q)$ spaces, Duke Math. J. 30 (1963), 129-142. MR 26 \#193.

9. - Fractional integration in Orlicz spaces. I, Trans. Amer. Math. Soc. 115 (1965), 300 328. MR 33 \# 3087.

10. R. C. Sharpley, Spaces $\Lambda_{\alpha}(X)$ and interpolation, J. Functional Analysis 11 (1972), 479513.

11. E. M. Stein, Singular integrals and differentiability properties of functions, Princeton Math. Ser., no. 30, Princeton Univ. Press, Princeton, N.J., 1970. MR 44 \#280.

12. L. Y. H. Yap, Some remarks on convolution operators and $L(p, q)$ spaces, Duke Math. J. 36 (1969), 647-658. MR 40 \# 3184. 
13. A. Zygmund, Trignometric series, 2nd rev. ed., Cambridge Univ. Press, New York, 1968. MR 38 \#4882.

14. Some points in the theory of trigonometric and power series, Trans. Amer. Math. Soc. 36 (1934), pp. 604,609.

Department of Mathematics, Oakland University, Rochester, Michigan 48063 